\title{
FITOMEJORAMIENTO PARTICIPATIVO DEL ARROZ DE SECANO EN NICARAGUA: METODOLOGÍAS, RESULTADOS Y LECCIONES APRENDIDAS 1
}

\author{
Gilles Trouche ${ }^{2}$, Lázaro Narváez-Rojas ${ }^{3}$, Zildghean Chow-Wong ${ }^{4}$, José Corrales-Blandón ${ }^{5}$
}

\begin{abstract}
RESUMEN
Fitomejoramiento participativo del arroz de secano en Nicaragua: metodologías, resultados y lecciones aprendidas. En varias regiones de Nicaragua, el cultivo de arroz de secano asume un papel importante para la seguridad alimenticia de las familias campesinas, además es fuente de ingresos para éstas. A partir del 2002, el CIRAD y el CIAT implementan en colaboración en Nicaragua un proyecto de investigación en fitomejoramiento participativo del arroz de secano dirigido a los pequeños y medianos productores.Este proyecto esta manejado en asociación con la Institución Nacional de Investigación Agropecuaria, varias ONGs e instituciones de extensión y organizaciones campesinas locales. En este trabajo, se describen la metodología general aplicada en el proyecto, los actores involucrados y los marcos de concertación entre los socios, las estrategias y métodos de evaluación y selección participativa aplicados y los principales resultados obtenidos al final de tres años. Esta experiencia deriva lecciones, con respecto al manejo de un proyecto de investigación asociando estrechamente fitomejoradores y productores, la selección de los socios, los métodos de trabajo, las competencias adquiridas por los actores y la diversidad genética explorada y valorizada, las que podrían ser utilizadas en la implementación de otros proyectos en este tema. Para la continuación del proyecto, los cambios de escala (scalingup), la organización de una producción descentralizada de semilla de calidad, el desarrollo de investigaciones en agronomía y la creación de un sistema permanente de evaluación y selección del arroz, reuniendo todos los actores de la cadena productiva, son los mayores retos.
\end{abstract}

Palabras clave: Fitomejoramiento, evaluación y selección participativa, arroz de secano, agricultura familiar.

\begin{abstract}
Rice participatory breeding in Nicaragua. In various regions of Nicaragua, upland rice production plays a major role for the food security of farmer's families and for generating incomes. Since 2002, CIRAD and CIAT develop together in Nicaragua a research project on participatory breeding of upland rice focused on small and medium-scale farmers. This project is managed in collaboration with the national agriculture research institution, NGOs, extension agencies and local farmers' organizations. In this paper, we described the general approach applied in the project, the participants involved, the research and dialogue frameworks, the breeding strategies and methods, and the main results obtained after three years of work. This experience provided lessons important for the development of research in close co-operation between breeders and farmers, the selection of partners, the working methods, and the exploration and valorization of genetic diversity, all of which may be useful for implementing future projects on the same topic. Future steps and main challenges of the project include scaling-up in the organization of a decentralized seed production system, the implementation of research activities in Agronomy and the creation of a permanent framework for rice breeding and evaluation which involves all the participants of the productive chain.
\end{abstract}

Key words: Plant breeding, participatory variety evaluation, unpland rice, family farming.

\footnotetext{
1 Recibido: 25 de febrero, 2006. Aceptado: 12 de junio, 2006.

2 CIAT-CIRAD, AA 6713, Cali, Colombia.

3 INTA, Programa Arroz, Centro Nacional Investigación Agropecuaria, Managua, Nicaragua.

4 CIAT-CIRAD, LM 172, Managua, Nicaragua.

5 INTA, Centro Experimental del Occidente, Posoltega, Nicaragua.
} 


\section{INTRODUCCIÓN}

En el marco de las relaciones institucionales CIAT-CIO ${ }^{6}$, el CIRAD ${ }^{7}$ y CIAT desarrollan en forma colaborativa en Nicaragua un proyecto de investigación en fitomejoramiento participativo de arroz y sorgo con el objetivo de responder mejor a las necesidades específicas de variedades mejoradas para los pequeños productores.

El fitomejoramiento participativo es una estrategia y un proceso de mejoramiento genético de los cultivos que generalmente busca desarrollar variedades apropiadas para los pequeños productores poco tecnificados en condiciones de producción marginales y/o con exigencias de calidad de grano específicas, donde el fitomejoramiento convencional no ha logrado proveer variedades mejoradas adecuadas. Para estas condiciones, el FP pretende desarrollar cultivares más estables y productivos, adaptados a las condiciones agro-ecológicas locales y con mejor aceptación para el autoconsumo y/o para la venta (Rosas et al. 2003). También, el FP propone a los agricultores una gama de cultivares de más amplia diversidad genética y contribuye a una cierta conservación dinámica de la biodiversidad local (Rosas et al. 2003). En América Central las primeras experiencias en FP tienen casi 10 años y han generado resultados importantes concerniente a variedades, conocimientos y capacidades colectivas (Hocdé 2006). Estos trabajos sobre FP estuvieron dirigidos casi exclusivamente a los rubros de frijol y maíz, y a pequeños productores de las zonas muy marginales (zonas de laderas y en altitud). El proyecto CIAT-CIRAD sobre arroz de secano en Nicaragua pretende ampliar las metas de aplicación del FP, no solo trabajando para los pequeños productores en sistemas de cultivo manuales, sino también para los productores medianos y tecnificados.

El arroz es un grano básico importante en varios países de América Central. El área total sembrada en arroz (secano + riego) en la región alcanza las 255 mil hectáreas. En Nicaragua el arroz de secano abarca

6 CIAT: Centro Internacional de Agricultura Tropical; CIO: Grupo de Instituciones francesas de investigación constituidas por el CIRAD, el INRA y el IRD (antiguamente denominado ORSTOM).

7 CIRAD: Centre de Coopération Internationale en Recherche Agronomique pour le Développement. aproximadamente 85 mil hectáreas y representa el 64\% del área total de producción (MAGFOR 2005). En condiciones de secano, los rendimientos son muy variables según la región y el sistema de producción, además dependen de las condiciones pluviométricas y fitosanitarias del año, variando de 2-3 t/ha en los sistemas manuales hasta 4-6 t/ha para los sistemas mecanizados en las zonas favorecidas.

Los principales problemas que enfrenta la producción de arroz de secano en Nicaragua son:

- Rendimientos bajos (promedio nacional de 1,4 t/ha) e irregulares (MAGFOR 2005);

- La escasez de variedades mejoradas adaptadas a los diversos sistemas de producción, particularmente para los sistemas poco tecnificados (Rizo y Parrales 2003) o las condiciones de secano no favorecido;

- Un control de malezas ineficiente;

- Problemas de sequía en las zonas arroceras del Pacífico;

- Presencia de suelos ácidos y baja luminosidad en las regiones del Atlántico;

- Plagas y enfermedades, principalmente piricularia y el complejo hongos-bacterias causando el manchado de grano y el vaneamiento de las panículas (INTA 2000);

- La calidad de grano del arroz nacional por lo general no reúne los requerimientos de la industria y por consiguiente compite difícilmente con el arroz importado (Oxfam 2005).

Es en este contexto que se inició en el año 2002 el proyecto de fitomejoramiento participativo de arroz de secano en Nicaragua para un periodo de cuatro años, en asociación con grupos de productores, ONGs, organizaciones campesinas, servicios de extensión rural e institutos de investigación agrícola.

El objetivo del presente trabajo es dar a conocer la metodología general aplicada y las etapas seguidas para implementar este proyecto, los tipos de actores involucrados y los marcos de concertación durante el periodo 2003-2005. Se presentan también las características y origen del germoplasma utilizado y los dispositivos y métodos de selección aplicados. Al final se presentan y se discuten los principales resultados generados por el proyecto, las lecciones aprendidas y las perspectivas. 


\section{MATERIALES Y MÉTODOS}

\section{Los actores involucrados}

Los investigadores CIAT-CIRAD presentaron y discutieron la propuesta del proyecto con diversas organizaciones que intervienen en apoyo a la agricultura familiar: i) ONGs e instituciones de extensión rural, ii) organizaciones de productores, iii) instituciones de investigación. La investigación agrícola pública, a través del Instituto Nicaragüense de Investigación Agropecuaria (INTA), está involucrada mediante estrechas colaboraciones con el programa nacional de arroz, los equipos regionales de investigación y extensión y mediante el intercambio de germoplasma. Estos contactos iniciales permitieron identificar una primera lista de socios interesados, conocer mejor sus métodos de trabajo y sus zonas de influencia, especificar sus intereses para participar en este proyecto. La literatura y los datos estadísticos existentes sobre la producción del arroz de secano en Nicaragua también fueron analizados.

Basándose en experiencias anteriores de investigación participativa ${ }^{8}$ y considerando el contexto particular de Nicaragua, desde el inicio los gestores del proyecto adoptaron el principio de trabajar, en la medida de lo posible con grupos de agricultores ya organizados en el marco de su comunidad.

En los sitios de intervención, se logró construir una colaboración múltiple entre las tres principales entidades participantes al proyecto, el equipo de investigación, las ONGs e instituciones de extensión y los grupos de productores, cuyas inter-relaciones se organizan en ambas direcciones.

\section{Selección de las zonas y sitios de trabajo}

El proyecto seleccionó sus zonas de intervención con base a cinco criterios, que no son jerárquicos:

- importancia de la producción de arroz de secano.

- producción mayormente asegurada por pequeñas y medianas explotaciones agrícolas.

8 Proyectos en Fitomejoramiento Participativo del frijol en la región Brunca en Costa Rica y del sorgo en Burkina Faso (Hocdé et al. 2002).
- presencia de grupos de productores localmente organizados.

- complementariedad geográfica y de objetivos, con el dispositivo de investigación nacional.

- presencia en la zona de uno o varios interlocutores (instituciones gobernamentales o no) dispuestos a apoyar técnicamente las actividades.

En cada zona pre-identificada, el equipo de investigación realizó, en un sitio propuesto por la institución local, un taller en donde se presentaron y discutieron los objetivos del proyecto con los grupos locales de productores. Estos talleres permitieron confirmar la selección de la zona y $\operatorname{los}_{\text {sitios }}{ }^{9}$ definitivos para implementar las actividades.

En total, cuatro sitios de intervención han sido identificados, ubicados en los departamentos de Chinandega, Rivas, Jinotega y en la Región Autónoma del Atlántico Norte (R.A.A.N.); además se realizaron actividades en San Dionisio (Matagalpa) por ser sitio de referencia para agricultura de laderas del CIAT en Nicaragua (Figura 1).

Chinandega: llanos del Pacífico, suelos de origen volcánico profundos y fértiles, clima cálido con pluviometría bastante favorable $(1.600-2.000 \mathrm{~mm})$, sistemas de cultivo en mayoría mecanizados e intensificados.

Belén (Rivas): colinas de poca altitud y llanos, zona seca (precipitaciones anuales $=800-1.200 \mathrm{~mm}$ ), sistemas de cultivo semi-mecanizados (siembra y manejo manual, cosecha mecanizada) o manuales.

Wiwilí, El Cúa, Bocay (Jinotega): valles del Río Coco y Río Bocay y otras zonas planas, precipitaciones abundantes (1.800-2.500 mm/año) y sistemas de cultivo manuales con bajos insumos.

Siuna (RAAN): planicie ligeramente ondulada, suelos moderadamente ácidos, precipitaciones abundantes (1.800-2.500 mm anuales) y baja luminosidad, sistemas de cultivo manuales con bajos insumos.

\footnotetext{
9 Sitio = zona espacial donde están establecidas las parcelas de ensayos; un sitio corresponde a una o varias localidades cercanas de un mismo departamento.
} 


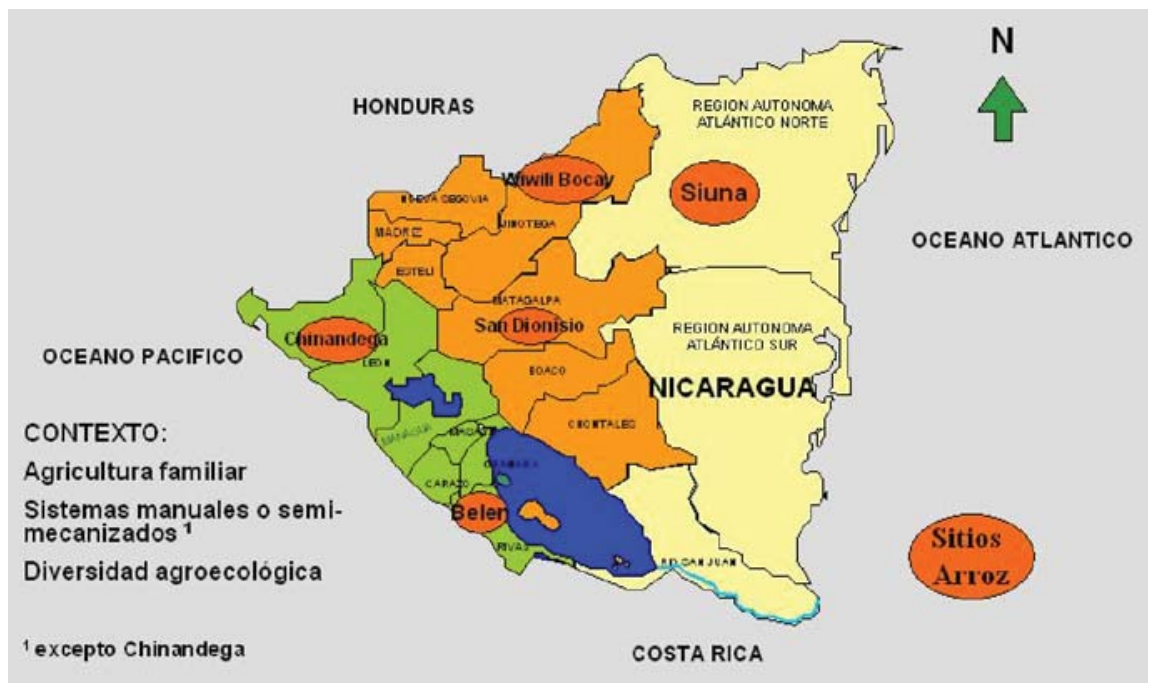

Figura 1. Sitios de intervención del proyecto fitomejoramiento participativo del arroz de secano en Nicaragua. 2003-2005.

\section{Implementación del proyecto}

En cada sitio, la ejecución de las actividades del proyecto siguió las etapas del proceso general presentado en la Figura 2. Se inició con la realización de un diagnóstico rápido encaminado a conocer mejor los sistemas de cultivo, las variedades utilizadas en los años anteriores y en la actualidad con sus características principales, los problemas y objetivos de producción y utilización, las formas de adquisición e intercambios de la

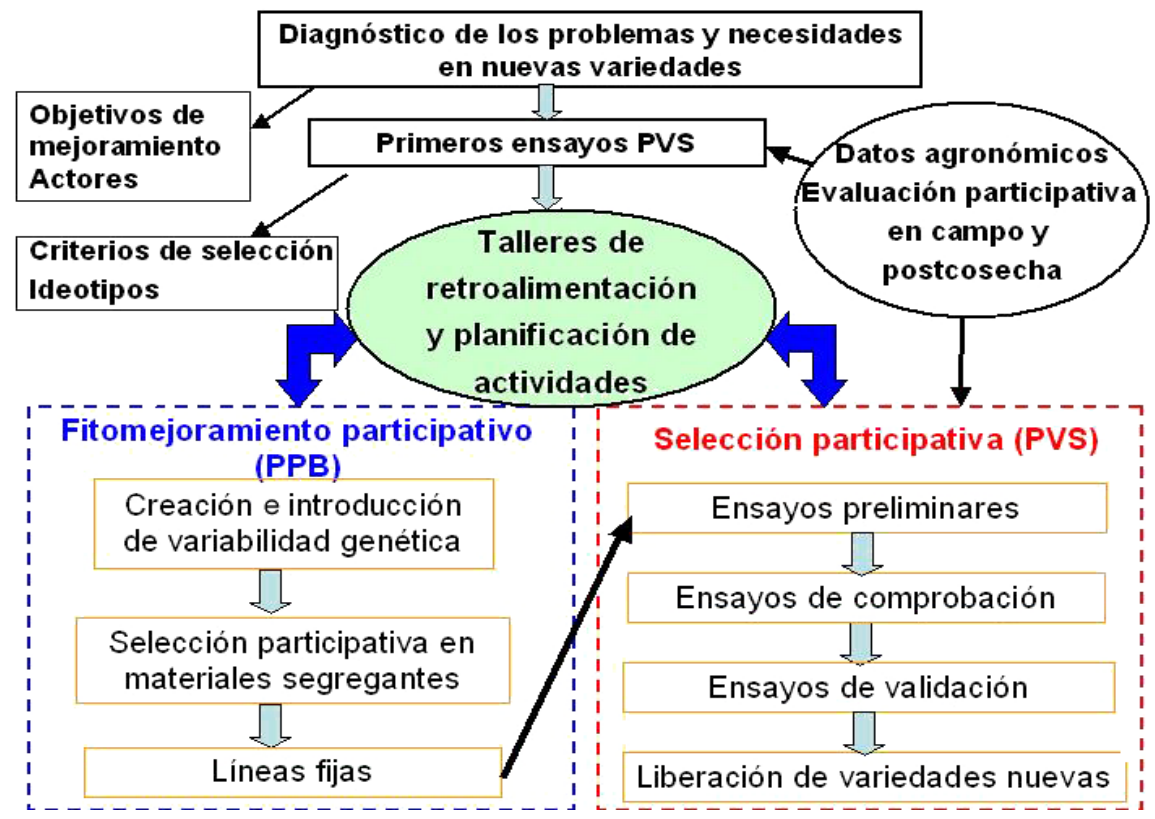

Figura 2. Etapas del proceso de fitomejoramiento participativo del arroz aplicado en el proyecto. 
semilla, entre otros, para finalmente determinar cuales son los objetivos de mejoramiento para la zona. Estos diagnósticos fueron realizados mediante talleres reuniendo un grupo representativo de productores (ras) de la comunidad. Para manejar estos talleres, varias herramientas del diagnóstico participativo fueron utilizados: la línea del tiempo (reseña histórica) del arroz en la zona, la descripción de los diferentes sistemas de cultivo, los inventarios de variedades.

Con base en la información generada por los diagnósticos, fueron establecidos en cada sitio los primeros ensayos de evaluación/selección participativa de líneas fijas y variedades (PVS en inglés) ${ }^{10}$, los cuales presentaron a los productores una gama diversificada de nuevos cultivares que puedan responder a los objetivos de mejoramiento definidos para la zona. En estos primeros ensayos y los siguientes, los investigadores o los técnicos de la institución de apoyo realizan las mediciones de las variables agronómicas comunes y los rendimientos; los cultivares también son evaluados por los productores (as) en el campo como en poscosecha para los aspectos de calidad de grano.
El análisis de los resultados agronómicos y las apreciaciones de los productores permitió a los investigadores, precisar los criterios de selección con sus respectivos grados de importancia y los ideotipos de los productores, e identificar entre los materiales genéticos presentados cuáles son los que se acercan más a estos ideotipos. A partir de estos resultados, las orientaciones globales (continuación de los ensayos PVS con germoplasma introducido y/o inicio de trabajos de selección participativa en materiales segregantes (PPB en inglés ${ }^{11}$ ), son discutidas y planificadas con los productores y los demás socios durante el taller de retroalimentación de resultados y planificación participativa de actividades (Figura 2).

En el Cuadro 1 se presentan los objetivos de mejoramiento inicialmente definidos con los productores a partir del diagnóstico y los primeros ensayos PVS en los sitios de intervención.

El Cuadro 2 suministra indicaciones cuantitativas de la dinámica del proceso durante los tres primeros años del proyecto.

Cuadro 1. Objetivos de mejoramiento identificados con los productores para los sitios de trabajo del proyecto. 2004.

\begin{tabular}{|c|c|c|}
\hline Sistema de cultivo & Sitios & Objetivos de mejoramiento \\
\hline $\begin{array}{l}\text { Tecnificado en condiciones de } \\
\text { secano favorecido }\end{array}$ & $\begin{array}{l}\text { Chinandega } \\
\text { (Pacífico Norte) }\end{array}$ & $\begin{array}{l}\text { Arquitectura de planta moderna: semi-enano }(60-80 \mathrm{~cm}) \text {, buen } \\
\text { macollamiento, hojas largas y delgadas semi-erectas. } \\
\text { Ciclos totales precoces ( } 90-110 \text { días) o intermedios }(110-130 \text { días) } \\
\text { Resistencia estable a piricularia, Rhizoctonia y manchado de grano } \\
\text { Alto potencial de rendimiento (superior a } 5 \mathrm{t} / \mathrm{ha}) \\
\text { Calidad industrial del grano }\end{array}$ \\
\hline $\begin{array}{l}\text { Semi-mecanizado o manual en } \\
\text { condiciones de secano menos } \\
\text { favorecidas }\end{array}$ & Belén (Pacífico Sur) & $\begin{array}{l}\text { Buen vigor de crecimiento inicial y altura intermedia }(90-120 \mathrm{~cm}) \\
\text { Tolerancia a sequía en fase vegetativa y/o ciclos precoces } \\
\text { Rendimientos mejorados }(3-4 \mathrm{t} / \mathrm{ha}) \text { y estables } \\
\text { Calidad de grano para autoconsumo y venta en el mercado nacional }\end{array}$ \\
\hline $\begin{array}{l}\text { Sistemas manuales en climas } \\
\text { sub-húmedos }\end{array}$ & $\begin{array}{l}\text { Siuna } \\
\text { Bocay-Wiwili } \\
\text { San Dionisio }\end{array}$ & $\begin{array}{l}\text { Alto vigor de crecimiento inicial y buena cobertura del suelo para } \\
\text { competir con las malezas } \\
\text { Porte adecuado para cosecha y aporreo manuales }(110-130 \mathrm{~cm}) \\
\text { Ciclos intermedios (110-130 días de la siembra a la madurez) } \\
\text { Resistencia estable a enfermedades (pyricularia, escaldado) } \\
\text { Tolerancia a suelos ácidos y alta nubosidad } \\
\text { Rendimientos mejorados en comparación a las variedades locales } \\
\text { Calidad de grano para autoconsumo y venta en mercado local }\end{array}$ \\
\hline
\end{tabular}

10 Participatory Varietal Selection.

ISSN: $1021-7444$
10 Participatory Plant Breeding. 
Cuadro 2. Evolución del número de agricultores involucrados, ensayos PVS establecidos y materiales segregantes en fase de selección en el proyecto FP arroz durante el período 2003-2005 en los cinco sitios de intervención en Nicaragua.

\begin{tabular}{lccc}
\hline \multicolumn{1}{c}{$\begin{array}{c}\text { Agricultores involucrados } \\
\text { y tipos de actividades }\end{array}$} & Año 1 (2003) & Año 2 (2004) & Año 3 (2005) \\
\hline Agricultores experimentadores (A/E) & 32 & 48 & 37 \\
Agricultores fitomejoradores & 16 & 15 & 10 \\
Ensayos PVS etapa 1 a & 21 & 8 & 4 \\
Ensayos PVS etapa 2 b & 18 & 0 & 38 \\
Ensayos PVS etapa 3 c & 0 & & 58 \\
Poblaciones y progenies segregantes & 65 & 250 & 587 \\
manejadas en fitomejoramiento participativo & 65 & \\
\hline
\end{tabular}

aensayos preliminares, bensayos de comprobación, censayos de validación (parcelas experimentales de $500 \mathrm{~m}^{2}$ )

\section{Papel y responsabilidades de las diferentes contra- partes}

Para cada sitio, un acuerdo informal, establecido entre el equipo de investigación, la ONG y/o la institución de extensión y/o la organización campesina (OC) y los grupos de productores, definen la distribución de las tareas y responsabilidades. Al inicio del proyecto, esta distribución de responsabilidades era como se indica a continuación:

\section{ONG, Institución de extensión u OC:}

- Propone las localidades y los grupos de productores voluntarios para involucrarse en el proyecto.

- Facilita la comunicación entre estos grupos y los investigadores del proyecto.

- Se encarga del establecimiento y el seguimiento continuo de los ensayos con los productores según un protocolo redactado por los investigadores.

- Anima los talleres de evaluación y selección de variedades (cuando los técnicos tienen un buen dominio de la metodología).

- Co-anima con los investigadores los talleres de retroalimentación de resultados a los productores y los de planificación de actividades.

\section{Agricultores:}

- Manejan los experimentos en sus parcelas ${ }^{12}$ según un protocolo entregado por los investigadores, conforme a lo discutido y negociado durante los talleres de planificación (número de repeticiones, tamaño de las parcelas, número e identidad de las variedades, entre otros). En estos experimentos se aplican las prácticas agronómicas comunes de la zona de producción.

- Realizan las evaluaciones de variedades tanto en campo como en postcosecha ${ }^{13}$.

\section{$\underline{\text { El Equipo de Investigación }}^{14}$ :}

- Suministra los protocolos, la semilla, los insumos (cuando los productores lo solicitan).

12 Suministran parcelas, tiempo y trabajo.

13 Para realizar estos experimentos y pruebas, los agricultores no reciben pago o indemnización, el proyecto solo suministra la semilla y algunos insumos (cuando éstos no lo pueden asumir) así como las muestras de arroz blanco y los ingredientes para las pruebas culinarias.

14 El Instituto Nicaragüense de Investigación Agropecuaria INTA y el proyecto CIAT-CIRAD. Estas dos entidades firmaron un contrato formal al inicio del proyecto, el cuál prevé, entre otros, el acceso a las estaciones experimentales de INTA para el equipo CIAT-CIRAD, un libre intercambio del germoplasma para los trabajos de experimentación, una utilización de toda la información generada por el proyecto (resultados, informes) por ambas instituciones y una definición conjunta de los derechos de propiedades de los materiales genéticos desarrollados por el proyecto. 
- Anima los talleres de evaluación y selección de variedades en campo (los dos primeros años).

- Analiza los resultados agronómicos de los ensayos y redacta los informes (memorias de los talleres e informes anuales de actividades).

- Prepara y co-anima los talleres de retroalimentación de resultados a los productores y los de planificación participativa de actividades.

- Capacita a los técnicos y a los productores sobre el manejo de los ensayos y los métodos de evaluación y selección participativa.

- Genera, en estación experimental, la diversidad genética necesaria para los futuros trabajos PPB.

\section{Dispositivos de concertación}

Los talleres anuales de retroalimentación y análisis de resultados y planificación de actividades, organizados en cada sitio de trabajo con todos los actores involucrados, constituyen el principal marco de concertación entre los participantes del proyecto. Durante estos talleres, se presenta y discute la síntesis de todos los resultados del ciclo agrícola anterior, además se planifican de manera consensuada las actividades del próximo ciclo agrícola.

En la etapa de retroalimentación de resultados, los investigadores presentan una síntesis de los cuatro tipos de resultados obtenidos (agronómicos, apreciación y selección de los cultivares por los agricultores en campo, pruebas culinarias y análisis de laboratorio) por todos los ensayos conducidos en el sitio, más eventualmente resultados de ensayos establecidos en otros sitios de agro-ecología similar. Al final de esta exposición los participantes discuten los resultados para tomar decisiones consensuadas relativas a los puntos siguientes:

- Cultivares a conservar o descartar en cada ensayo.

- Ajustes sobre el manejo de los ensayos, los talleres de selección participativa.

- Nuevos temas de experimentación y temas de capacitación.

- Recomendaciones para corregir los inconvenientes presentados y superarlos éxitosamente.

\section{Germoplasma}

Como principio inicial, el proyecto decidió utilizar una amplia diversidad genética. Por lo tanto se planteó la hipótesis que, entre la variabilidad genética de las líneas avanzadas (genéticamente fijas) recientemente generadas por el CIAT y el CIRAD en Colombia, en particular el germoplasma derivado de selección recurrente (Châtel et al. 2003) y de cruces inter-específicos (Martínez et al. 1998), era posible identificar materiales genéticos que se adaptaran a las condiciones agro socioeconómicas de los diferentes sistemas de producción del arroz de secano existentes en Nicaragua y otros países de América Central. El Cuadro 3 presenta los orígenes y fondos genéticos de las líneas y variedades utilizadas en esta fase PVS del proyecto.

Sin embargo, a partir del 2003 y sobre todo en 2004, una vez bien identificados los criterios de selección y las preferencias de los productores, asimismo mejor organizados los dispositivos de trabajo con los socios, se iniciaron esquemas de fitomejoramiento participativo (PPB) a partir de poblaciones sintéticas de amplia diversidad genética y adaptación o progenies derivadas de éstas, de poblaciones F2 derivadas de cruces entre poblaciones y buenas variedades locales y finalmente de poblaciones sintéticas de sitio-específico creadas en Nicaragua (Cuadro 3).

\section{Estrategias y métodos de selección}

Dos estrategias de selección participativa son aplicadas en este proyecto:

- Selección participativa entre líneas avanzadas (fijas) y variedades (PVS).

- Creación participativa de nuevas líneas en materiales segregantes o fitomejoramiento participativo en el sentido estricto (PPB).

La primera estrategia pretende aprovechar el material vegetal ya existente y disponible con el fin de dar resultados a corto plazo (nuevas variedades rápidamente adoptadas por los productores) y con un costo menor (Witcombe et al. 1996). La segunda estrategia necesita más recursos técnicos y financieros (en términos de creación de variabilidad genética, organización del trabajo entre los socios y capacitación de los productores) y genera resultados a mediano y largo plazo. 
Cuadro 3. Orígenes genético y geográfico del germoplasma de arroz utilizado por el proyecto entre 2003 y 2005.

\begin{tabular}{|c|c|c|c|}
\hline Método & Origen genético & $\begin{array}{l}\text { Institución y } \\
\text { país de origen }\end{array}$ & $\begin{array}{l}\text { Número de } \\
\text { materiales }\end{array}$ \\
\hline \multirow[t]{6}{*}{ PVS } & Líneas japónica procedentes de mejoramiento convencional (pedigrí) & $\begin{array}{l}\text { CIRAD-CIAT } \\
\text { Colombia }\end{array}$ & 27 \\
\hline & Líneas derivadas de cruces interespecíficos & CIAT Colombia & 118 \\
\hline & $\begin{array}{l}\text { Líneas japónica derivadas de selección recurrente (Poblaciones PCT-4 y } \\
\text { PCT-11) }\end{array}$ & $\begin{array}{l}\text { CIRAD-CIAT } \\
\text { Colombia }\end{array}$ & 176 \\
\hline & Líneas indica derivadas de esquemas backcross para resistencia a piricularia & CIAT Colombia & 50 \\
\hline & $\begin{array}{l}\text { Variedades de amplia adaptación geográfica con resistencia durable }{ }^{1} \text { a } \\
\text { piricularia }\end{array}$ & $\begin{array}{l}\text { CIRAD y otras } \\
\text { fuentes }\end{array}$ & 10 \\
\hline & Otras líneas experimentales indica & INTA & 20 \\
\hline \multirow[t]{2}{*}{ PPB } & Poblaciones y líneas segregantes introducidas (2003 y 2004) & $\begin{array}{l}\text { CIRAD-CIAT } \\
\text { Colombia }\end{array}$ & $\begin{array}{l}4 \text { poblaciones }+365 \\
\text { líneas } S_{2}-S_{5}\end{array}$ \\
\hline & Variabilidad genética creada en Nicaragua (2004 y 2005) & $\begin{array}{l}\text { CIRAD-CIAT/ } \\
\text { INTA }\end{array}$ & $\begin{array}{c}3 \text { cruces }+3 \\
\text { poblaciones de } \\
\text { sitio-específico }\end{array}$ \\
\hline
\end{tabular}

1 Una estrategia de selección para la resistencia durable a piricularia aplicada por el CIRAD en sus programas de mejoramiento genético de arroz de secano ha sido el uso de la resistencia parcial oligogénica controlada por un conjunto de genes menores independientes (Vales 1987); la resistencia durable, de tipo parcial, de estas variedades CIRAD fue comprobada durante más de 10 años en diferentes países y regiones de Africa y América Latina (Vales 2002, Comunicación personal ${ }^{15}$ ) y se ha verificado en Nicaragua.

La evaluación y selección participativa de las nuevas líneas o variedades es manejada en dos etapas: i) evaluación en campo en una fase cercana a la madurez fisiológica y selección de las variedades preferidas, ii) evaluación en poscosecha de la calidad de grano de las mejores variedades mediante pruebas culinarias. La evaluación en campo es conducida según un método desarrollado en Burkina Faso-África (Vom Brocke et al. 2003) y adaptado en Nicaragua (Trouche et al. 2003), basado en la utilización de los criterios de selección propios de los productores de la comunidad; estos criterios son previamente identificados, discutidos y priorizados durante una sesión de trabajo conducida en el mismo sitio, antes de realizar la evaluación en campo. Organizados en pequeños grupos de tres a cinco personas o de manera individual, los productores participantes evalúan todos los cultivares, en dos o tres repeticiones del ensayo, esto conforme a los cuatro criterios de selección de mayor importancia y el valor general de la variedad, y los califican según una escala

15 VALES, M. 2002. Variedades con resistencia durable a piricularia (entrevista). CIAT, Cali, Colombia. Correo electrónico: michel.vales@cirad.fr de cuatro niveles: malo, regular, bueno, excelente. A partir de esta evaluación, cada grupo indica las variedades preferidas, las que quieren seleccionar para el ciclo siguiente (generalmente se aplica una intensidad de selección entre 20 y $30 \%$ ).

El fitomejoramiento participativo (PPB) se maneja con una muestra más reducida de productores voluntarios y expertos. Los objetivos de selección, y a veces los progenitores de los cruzamientos a realizar, son co-definidos entre los investigadores y los productores. Los cruzamientos y las generaciones sin selección son ejecutados por el fitomejorador durante la época seca (con riego) en la estación experimental del INTA en Sébaco. Las generaciones de selección son manejadas en los sitios metas, ya sea en estación de investigación (Centro Experimental del Occidente en la zona Chinandega) o en una finca experimental (Siuna y San Dionisio). Dos estrategias de selección están siendo aplicadas:

\section{Estrategia a mediano plazo}

- Selección pedigrí a partir de poblaciones o líneas segregantes introducidas. 
Estrategia a mediano y largo plazo

- Creación de poblaciones de base genética ampliada (contando con el aporte de variabilidad de seis a ocho progenitores) para responder a objetivos de mejoramiento específicos.

- Mejoramiento de las poblaciones utilizando el método de selección recurrente.

- Selección de plantas dentro de las poblaciones y desarrollo de líneas fijas mediante el método de selección pedigrí.

\section{Evolución del proceso y de los dispositivos}

A medida que se ha desarrollado el proyecto y por los mecanismos de concertación entre los actores, algunas evoluciones y ajustes han sido incorporados a los dispositivos iniciales, igualmente algunas novedades se han introducidos. Estas adaptaciones se realizan con el objetivo de mejorar la eficiencia y la estabilidad del dispositivo, dar más responsabilidad y capacidad técnica a los productores experimentadores y mejoradores, y a sus organizaciones y liberar tiempo para los investigadores ${ }^{16}$. Mientras tanto, los métodos de trabajo son afinados, lo que corresponde al segundo objetivo del proyecto.

Durante los talleres de evaluación y selección en campo, los productores expresan a veces nuevas ideas para incluir nuevos criterios de selección (por ejemplo, la uniformidad de maduración en Chinandega, el vigor de crecimiento para competir con las malezas en Siuna), o ajustar la clasificación de los criterios más importantes de un ciclo de cultivo a otro (por ejemplo la resistencia al complejo manchado de grano en Chinandega). Durante los talleres anuales de retroalimentación y análisis de los resultados, aparecen a veces contradicciones entre las conclusiones de los investigadores y las de los productores; de eso surgen discusiones, argumentaciones y debates ${ }^{17}$.

\footnotetext{
16 Para que puedan dedicar más tiempo en su primera tarea, es decir crear variabilidad y desarrollar nuevo gemoplasma.

17 Por ejemplo, en 2005 la línea CT 11231 fue calificada de excelente y ampliamente seleccionada por los productores en los sitios de Rivas y Siuna pero no esta entre las mejores en rendimiento final. Lo contrario sucedió con INTA Dorado en la zona de de Wiwilí, donde presentó los mayores rendimientos pero por su bajo porte y ciclo más tardío fue rechazada por los productores.
}

Resultados más relevantes obtenidos después de tres ciclos agrícolas

Los resultados obtenidos después de tres ciclos agrícolas son múltiples y conciernen cuatro tipos de productos:

- Germoplasma

- Definición de métodos

- Producción de conocimientos

- Aprendizaje y fortalecimiento de capacidades de los actores

\section{Germoplasma}

Los materiales genéticos seleccionados y/o generados por medio del proyecto pueden ser divididos en tres categorías:

Nuevos cultivares que se adaptan directamente a los sistemas de cultivo existentes

Los nuevos cultivares que se adaptan directamente a los sistemas de cultivo existentes han sido seleccionados por los agricultores, tomando en cuenta la productividad, el porte de planta, el ciclo adecuado para la zona, la resistencia a las enfermedades, la apariencia y calidad del grano y otros criterios menores.

En la zona de Jinotega, las variedades IRAT 364, IRAT 366 e IRAT 362, son las más aceptadas por los productores entre las diez variedades evaluadas en la zona desde 2003, y pasaron a la fase de validación durante el ciclo agrícola 2005. Considerando los resultados obtenidos en cinco localidades de la zona, las tres variedades superaron en $34-55 \%$ en rendimiento a la variedad del productor (Cuadro 4). Entre estas, IRAT 362 es la que tiene la mejor calidad industrial de grano (Cuadro 4). En las evaluaciones en postcosecha y pruebas culinarias que se realizaron en tres sitios en 2004, los productores y productoras han preferido IRAT 362 e IRAT 366, comparado a IRAT 364 (Cuadro 4).

Para los sistemas manuales de la zona de Belén (Rivas), entre las siete líneas más promisorias seleccionadas por los productores en 2004, la línea CT 15944-104-3-3 mostró las mejores características agronómicas (porte de planta, tolerancia al acame), obtuvo el mejor 
Cuadro 4. Resultados agronómicos y apreciación de los productores en campo y calidad de grano de tres variedades en ensayos de validación en Wiwilí-El Cúa (Jinotega), 2005.

\begin{tabular}{|c|c|c|c|c|c|c|c|c|c|}
\hline Variedades & $\begin{array}{c}\text { Días a } \\
\text { floración }\end{array}$ & $\begin{array}{c}\text { Altura } \\
\text { planta } \\
(\mathrm{cm})\end{array}$ & $\begin{array}{c}\text { Rendimiento } \\
\text { grano } \\
\text { (kg/ha) }\end{array}$ & $\begin{array}{c}\text { Incremento } \\
\text { versus } \\
\text { testigo \% }\end{array}$ & IAP1 & $\begin{array}{l}\text { Rendimien- } \\
\text { tos industria- } \\
\text { les }(\%)^{2}\end{array}$ & $\begin{array}{c}\text { Centro } \\
\text { blanco } \\
(\%)\end{array}$ & $\begin{array}{c}\text { Textura } \\
\text { del arroz } \\
\text { cocido }^{3}\end{array}$ & $\begin{array}{c}\text { Sabor } \\
\text { del arroz } \\
\text { cocido }^{3}\end{array}$ \\
\hline IRAT 364 & 89 & 150 & 3.314 & +55 & 3,0 & $69,2(53)$ & 8,4 & Suelto & Bueno \\
\hline IRAT 366 & 76 & 130 & 3.111 & +45 & 3,2 & $67,4(55)$ & 7,2 & Suelto & Excelente \\
\hline IRAT 362 & 86 & 123 & 2.877 & +34 & 2,9 & $67,5(62)$ & 0,1 & Suelto & Excelente \\
\hline Variedad productor & 99 & 153 & 2.140 & & 2,1 & & & Intermedio & Excelente \\
\hline Media & & & 2.861 & & & & & & \\
\hline C.V. $(\%)$ & & & 23 & & & & & & \\
\hline$F$ variedad & & & NS & & & & & & \\
\hline
\end{tabular}

1 IAP: Índice de apreciación general de la variedad por los productores en campo con respecto a los cuatro criterios de selección más importantes y al valor general (multiplicado por un coeficiente dos) según la siguiente escala: $1=$ malo, $2=$ regular, $3=$ bueno y $4=$ excelente; valor promedio de cinco ensayos PVS conducidos en esta zona entre 2003 y 2004.

2 Rendimiento en arroz oro, entre paréntesis rendimiento en grano entero.

3 Resultados de la apreciación de los productores (as) en las pruebas culinarias realizadas en tres localidades de la zona de Wiwilí-Bocay (2004).

rendimiento promedio y tuvo una muy buena apreciación por los productores en campo (Cuadro 5). A pesar de condiciones edafo-climáticas muy diferentes, esta línea se comportó también muy bien en los sistemas manuales de la zona de Siuna en $2005^{18}$.

Al igual que en estos dos sitios, los trabajos de evaluación y selección participativa "rápidamente descentralizados" han permitido identificar en los demás sitios, líneas y variedades con alta aceptación de los productores y mayores rendimientos, comparados a la variedad local o comercial más cultivada en esta zona. Sin embargo, las ganancias de rendimiento logradas difieren entre los sitios, siendo mayores las obtenidas en los sistemas manuales, comparadas a los sistemas tecnificados o semi-tecnificados (Cuadro 6).

\section{Cultivares precoces ofreciendo nuevas oportu- nidades de producción}

Entre las variedades identificadas por el proyecto, se debe de mencionar el caso de líneas extra-precoces (menos de 90 días de ciclo total) que ofrecen nuevas

18 Primer lugar en la selección de los productores y tercer lugar en rendimiento. alternativas de producción del arroz en las regiones del Pacífico de Nicaragua. Por sus ciclos muy cortos, se pueden sembrar al inicio de la segunda época lluviosa (postrera) y por consiguiente escapan del problema de déficit hídrico que ocurre frecuentemente durante el veranillo ${ }^{19}$, estrés que las variedades de 110-120 días comúnmente utilizadas deben sufrir en las zonas de secano del Pacífico. Además permiten realizar una rotación leguminosa de ciclo corto con arroz durante el mismo ciclo agrícola. Entre las líneas más promisorias experimentadas desde 2003, dos líneas derivadas de la población PCT-4 se encuentran en fase de validación y, con base en los resultados obtenidos en dos sitios en 2005, tres nuevas líneas van a seguir este proceso en 2006 (Cuadro 7). Es interesante notar que estas cinco líneas más promisorias provienen de tres esquemas diferentes de mejoramiento genético del arroz de secano: PCT-4\SA $1 \backslash 1>516$, PCT-4\SA $\backslash 1 \backslash 1>982$ y PCT4\SA\1\1>1479 (selección recurrente, CIRAD/CIATColombia), WAB 758-1-1-HB-4 (selección a partir de cruces interespecíficos, WARDA-África) y CT 118913-3-3-M-5-1-1-M (selección pedigrí convencional, CIRAD/CIAT-Colombia).

19 El veranillo o canícula es el nombre localmente utilizado para referirse al periodo relativamente seco ubicado entre el 15 de julio y el 15 de agosto, entre las épocas de primera (mayo a julio) y postrera (agosto a noviembre). 
Cuadro 5. Síntesis de los resultados agronómicos y preferencias de los productores en la zona de Belén (Rivas), 2004-2005.

\begin{tabular}{|c|c|c|c|c|c|}
\hline Líneas & $\begin{array}{c}\text { Días a } \\
\text { floración }\end{array}$ & $\begin{array}{c}\text { Altura } \\
\text { planta }(\mathbf{c m})\end{array}$ & $\begin{array}{c}\text { Rendimiento } \\
\text { grano } \\
\text { promedio }(\mathrm{kg} / \mathrm{ha})\end{array}$ & $\%$ testigo & $\begin{array}{c}\text { Frecuencia } \\
\text { selección } \\
\text { productores }^{1}\end{array}$ \\
\hline CT 15944-10-4-3-3 & 79 & 121 & $4.268 \mathrm{a}^{2}$ & 112 & 0,67 \\
\hline CT 15944-10-4-3-1 & 80 & 128 & $4.119 \mathrm{a}$ & 108 & 0,3 \\
\hline CT 15944-10-18-1-1 & 80 & 136 & $3.603 \mathrm{ab}$ & 94 & 0,67 \\
\hline WAB 894-B-5A2.1-4 & 80 & 99 & $3.093 \mathrm{ab}$ & 81 & 0,34 \\
\hline CT 15030-26-3-3-3 & 83 & 118 & $2.802 \mathrm{ab}$ & 73 & 0,17 \\
\hline WAB 759-54-2-3-HB-1 & 79 & 144 & $2.796 \mathrm{ab}$ & 73 & 0,33 \\
\hline CT 11231-2-2-1-3-M-4-5-1M & 79 & 102 & $2.697 \mathrm{ab}$ & 71 & 1,00 \\
\hline Variedad Comercial & 95 & 109 & $3.819 \mathrm{ab}$ & 100 & 0,34 \\
\hline Media del ensayo & & 3.399 & & & \\
\hline C.V. $(\%)$ & & $28^{3}$ & & & \\
\hline F Ambiente & & $*$ & & & \\
\hline F Genotipo & & $* *$ & & & \\
\hline F Ambiente x Genotipo & & $* *$ & & & \\
\hline \multicolumn{6}{|c|}{$\begin{array}{l}1 \text { Frecuencia promedia de selección de las líneas por los productores durante la evaluación en campo realizada en } \\
\text { dos de los tres ensayos } \\
*, * * \text { : valor de F significativo al } 5 \text { y } 1 \% \text {, respectivamente; } \\
2 \text { Los tratamientos con la misma letra no son significativamente diferentes según la prueba de Tukey al } 5 \% \text {. } \\
3 \text { El alto valor del coeficiente de variación para el rendimiento en grano se debe a heterogeneidades de terreno en uno } \\
\text { de los sitios de experimentación, lo que es común para ensayos establecidos en finca de productores. }\end{array}$} \\
\hline
\end{tabular}

Cuadro 6. Incrementos en rendimiento con relación a la variedad comercial obtenidos con las mejores líneas procedentes de los viveros introducidos en cuatro sitios de trabajo del proyecto. 2003.

\begin{tabular}{lccc}
\hline Sitio & $\begin{array}{c}\text { Rendimiento promedio de } \\
\text { las cinco mejores líneas (kg/ha) }\end{array}$ & $\begin{array}{c}\text { Incremento de rendimiento } \\
\text { de la mejor línea comparado } \\
\text { a la variedad comercial testigo } \mathbf{1}^{\mathbf{s}}\end{array}$ & $\begin{array}{c}\text { Número de líneas que } \\
\text { superan en rendimiento a } \\
\text { la variedad testigo }^{\mathbf{1}}\end{array}$ \\
\hline Belén & 3.577 & $+12 \%$ versus INTA N-1 & 2 \\
Siuna & 3.661 & $+26 \%$ versus Raizora Amarilla & 7 \\
San Dionisio & 6.538 & $+29 \%$ versus IRAT 366 & 6 \\
Chinandega (líneas Japónica) & 4.590 & $+7 \%$ versus INTA N-1 & 2 \\
\hline
\end{tabular}

${ }^{1}$ Resultados promedios obtenidos a partir del análisis de varianza combinado de varios ensayos establecidos en cada sitio entre 2004 y 2005: Belén (tres ensayos), Siuna (un ensayo), San Dionisio (dos ensayos), Chinandega Japónica (tres ensayos).

\section{Para el futuro, una amplia diversidad de líneas desarrolladas con la participación directa de los agricultores mejoradores}

Para el mediano y largo plazo, las actividades en PPB están generando nuevas líneas de arroz abarcando una diversidad genética ampliada (Cuadro 8).

\section{Métodos}

- Organizar una investigación FP en colaboración con diferentes socios.

- Definición consensuada de objetivos de selección.

- Método de evaluación y selección participativa de variedades basándose en los criterios de selección de los productores (Trouche y Hocdé 2006). 
Cuadro 7. Resultados agronómicos y apreciación de los productores de las mejores nuevas líneas precoces evaluadas en la zona del Pacífico en 2005.

\begin{tabular}{|c|c|c|c|c|c|c|}
\hline Línea & $\begin{array}{c}\text { Días a } \\
\text { floración } 1\end{array}$ & $\begin{array}{c}\text { Altura de } \\
\text { planta }(\mathrm{cm})^{1}\end{array}$ & $\begin{array}{l}\text { Rendimiento } \\
\text { grano }(\mathrm{kg} / \mathrm{ha})^{1}\end{array}$ & $\begin{array}{c}\% \\
\text { testigo }\end{array}$ & IAP1 & $\begin{array}{c}\text { Frecuencia } \\
\text { selección } \\
\text { productores }\end{array}$ \\
\hline 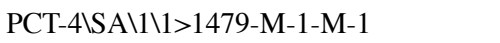 & 57 & 84 & 2.858 & 118 & 3,1 & 0,88 \\
\hline WAB 758-1-1-HB-4 & 57 & $92 \mathrm{~s}$ & 2.838 & 117 & 3,1 & 0,71 \\
\hline CT 11891-3-3-3-M-5-1-1-M & 57 & 82 & 2.700 & 111 & 2,1 & 0,25 \\
\hline 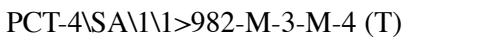 & 56 & 80 & 2.430 & 100 & 2,3 & 0,25 \\
\hline 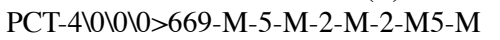 & $60 \mathrm{~s}$ & $94 \mathrm{~s}$ & 2.391 & 98 & 2,6 & 0,29 \\
\hline 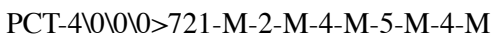 & 59 & 88 & 2.328 & 96 & 2,5 & 0,42 \\
\hline 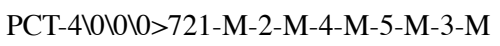 & 59 & $93 \mathrm{~s}$ & 2.287 & 94 & 2,9 & 0,50 \\
\hline Media del ensayo & 58 & 85 & 2.272 & & & \\
\hline C.V. $(\%)$ & 3,5 & 7,1 & 19,6 & & & \\
\hline F línea & $* *$ & $* *$ & * & & & \\
\hline F sitio & $* *$ & $* *$ & $* *$ & & & \\
\hline F sitio x línea & $* *$ & $*$ & $*$ & & & \\
\hline
\end{tabular}

${ }^{1}$ Promedio de dos ensayos establecidos en 2005 (Chinandega y Masaya).

*,**: Valor de F significativo al 5 y $1 \%$, respectivamente.

s: Significativamente superior al testigo según la prueba estadística de Dunnett al $5 \%$.

Cuadro 8. Progenies seleccionadas por los agricultores mejoradores y los investigadores en los sitios de Chinandega (Región del Pacífico) y Siuna (Región del Atlántico) en 2005.

\begin{tabular}{|c|c|c|c|c|c|}
\hline \multirow[t]{2}{*}{ Sitio } & \multirow{2}{*}{$\begin{array}{l}\text { Cruces o poblaciones fuentes } \\
\text { de diversidad }\end{array}$} & \multicolumn{2}{|c|}{ Chinandega } & \multicolumn{2}{|c|}{ Siuna } \\
\hline & & $\begin{array}{l}\text { Número de pro- } \\
\text { genies evaluadas }\end{array}$ & $\begin{array}{l}\text { Número de plantas } \\
\text { seleccionadas }\end{array}$ & $\begin{array}{l}\text { Número de proge- } \\
\text { nies evaluadas }\end{array}$ & $\begin{array}{l}\text { Número de plantas } \\
\text { seleccionadas }\end{array}$ \\
\hline \multirow[t]{11}{*}{ Chinandega } & Cruces & & & & \\
\hline & PCT-18/INTA N-1 & 57 & 26 & 1 & I \\
\hline & PCT-18/Raizora Am. & I & I & 91 & 66 \\
\hline & PCT-18/Criolla Siuna & l & / & 109 & 36 \\
\hline & Poblaciones & & & & \\
\hline & PCT-4 & 42 & 51 & 26 & 28 \\
\hline & PCT-11 & 19 & 12 & 57 & 79 \\
\hline & PCT-18 & 34 & 25 & l & l \\
\hline & CNA-7 & 0 & 0 & 57 & 7 \\
\hline & PCTNIC-1 & $1 \mathrm{~F} 2$ & 136 & I & l \\
\hline & TOTAL & 153 & 250 & 340 & 216 \\
\hline
\end{tabular}

- Método de creación participativa de líneas a partir de cruces simples o de poblaciones sintéticas de más amplia diversidad genética, utilizando el método de selección pedigrí.

- Método de mejoramiento participativo de estas poblaciones por el método de selección recurrente

Los esquemas de fitomejoramiento participativo utilizando los métodos pedigrí y recurrente han sido definidos y se están aplicando en tres sitios.

\section{Conocimientos}

- Mejor conocimiento, tanto para los investigadores como los técnicos y agricultores, de las características y problemas de los sistemas de cultivo del arroz de secano en Nicaragua.

- Variedades tradicionales caracterizadas por su valor agronómico o calidad de grano ${ }^{20}$.

\footnotetext{
20 Bluebonnet y Carbonet en Rivas, Raizora Amarillo en Siuna.
} 
- Variedades mejoradas modernas para las cuales las zonas y condiciones de adaptación están mejor definidas ${ }^{21}$.

- Criterios de selección de los productores identificados y priorizados según la zona o el sistema de cultivo.

- Definición de ideotipos por los agricultores en interacción con los investigadores.

\section{Aprendizaje y fortalecimiento de capacidades de los actores}

Capacidades adquiridas por los productores

- Capacidad de definir objetivos de mejoramiento genético para un cultivo.

- Determinación consensuada de los criterios de adaptación y calidad para una variedad de arroz.

- Organización colectiva y capacidades individuales para establecer ensayos, manejarlos, medir datos agronómicos, evaluar y seleccionar variedades, interpretar los resultados de experimentos y discutir con los investigadores.

- Organización colectiva de toma de decisiones entre productores y con los investigadores.

\section{Competencias adquiridas por los fitomejoradores}

- Incorporación de nuevos criterios de selección identificados por los productores en los futuros esquemas de mejoramiento del arroz.

- Capacidad de trabajar en equipo con diferentes socios y con agricultores.

- Capacidad para iniciar y manejar un proyecto FP en su globalidad.

- Prepararación y manejo de talleres de evaluación en campo y poscosecha, de retroinformación de resultados y de planificación de actividades con

21 Ejemplo de la variedad INTA Dorado. productores. Esto requiere nuevas competencias para los fitomejoradores quienes deben adquirir o reforzar su dominio de métodos y herramientas de comunicación con los agricultores.

- Cuestiones e ideas nuevas para manejar un esquema de mejoramiento genético de manera diferente (proceso, criterios, índice de selección, análisis de los datos).

\section{DISCUSIÓN}

El proyecto FP arroz desarrollado en Nicaragua introdujo varios cambios importantes en comparación al programa de fitomejoramiento convencional del arroz de secano en Nicaragua: la descentralización de los trabajos de fitomejoramiento y evaluación de líneas hacia las zonas de producción metas es más importante y ocurre más temprano en el esquema general de desarrollo de las variedades, los productores participan en las etapas de selección en materiales segregantes y en las fases iniciales de evaluación de líneas, se crearon en las zonas de trabajo mecanismos de concertación y discusión entre los investigadores, los técnicos y los productores, para tomar en cuenta las necesidades y las solicitudes de los pequeños productores.

\section{Lecciones}

Tres años de experiencia en la implementación del proyecto nos permiten evaluar la pertinencia de las orientaciones iniciales. La evaluación y selección participativa descentralizada para responder a diferentes condiciones y objetivos de producción, manejado de manera muy pragmática, permitió identificar una nueva diversidad de cultivares de altas calidades más aceptados por los productores, comparados a las variedades comerciales existentes. Los primeros materiales seleccionados con los productores mediante la estrategia PVS ya entraron en validación en el 2005 (seis variedades) o entrarán en esta fase en el 2006 (Cuadro 9). La elección inicial de conducir las actividades FP con grupos de productores localmente organizados y apoyados por una institución local "fuerte" ha sido pertinente: esto grupos han obtenido resultados más satisfactorios en menos tiempo, comparados a los grupos constituidos al inicio del proyecto, los cuales están 
Cuadro 9. Características, rendimientos promedio y apreciaciones de los productores de los cultivares de arroz más promisorios identificados en el proyecto FP en Nicaragua. 2005.

\begin{tabular}{|c|c|c|c|c|c|c|}
\hline Cultivar & Tipo genético & Principales características & $\begin{array}{l}\text { Sistemas de pro- } 1 \\
\text { ducción y zona } \\
\text { de adaptación }\end{array}$ & $\begin{array}{l}\text { Rendimiento } \\
\text { promedio } \\
\text { (kg/ha) }\end{array}$ & IAP & $\begin{array}{l}\text { Frecuencia } \\
\text { de selección } \\
\text { por los pro- } \\
\text { ductores }\end{array}$ \\
\hline IRAT 366 & Japónica & $\begin{array}{l}\text { Buen rendimiento, buen porte para sistemas manuales, } \\
\text { tallos gruesos y resistente al acame, rusticidad y resis- } \\
\text { tencia durable a piricularia. }\end{array}$ & $\begin{array}{l}\text { Sistemas manua- } \\
\text { les Jinotega }+ \\
\text { Atlántico Norte }\end{array}$ & $\begin{array}{l}3.111^{1} \\
(2.140)\end{array}$ & $\begin{array}{l}3,222^{2} \\
(2,12)\end{array}$ & $\begin{array}{c}0,80^{2} \\
(0)\end{array}$ \\
\hline IRAT 362 & Japónica & $\begin{array}{l}\text { Excelente calidad industrial de grano, altura interme- } \\
\text { dia y resistencia durable a piricularia. }\end{array}$ & $\begin{array}{l}\text { Sistemas manua- } \\
\text { les Jinotega }\end{array}$ & $\begin{array}{l}2.877^{1} \\
(2.140)\end{array}$ & $\begin{array}{l}2,922 \\
(2,12)\end{array}$ & $\begin{array}{c}0,54^{2} \\
(0)\end{array}$ \\
\hline $\begin{array}{l}\text { CT } 15944- \\
10-4-3-3\end{array}$ & $\begin{array}{l}\text { 7/8 Japónica } \\
1 / 8 \text { O. glabe- } \\
\text { rrima }\end{array}$ & $\begin{array}{l}\text { Alto vigor de crecimiento y macollamiento, alto poten- } \\
\text { cial de rendimiento, buena calidad de grano. }\end{array}$ & $\begin{array}{l}\text { - Sistemas manua- } \\
\text { les Rivas y Siuna }\end{array}$ & $\begin{array}{l}4.268^{3} \\
(3.816)\end{array}$ & $\begin{array}{c}2,75^{3} \\
(2,3)\end{array}$ & $\begin{array}{l}0,673 \\
(0,34)\end{array}$ \\
\hline $\begin{array}{l}\text { WAB } 894-B- \\
5 \text { A2.1-4 }\end{array}$ & $\begin{array}{l}\text { 3/4 Japónica } \\
1 / 4 \text { O. glabe- } \\
\text { rrima }\end{array}$ & $\begin{array}{l}\text { Semi-enano, buen macollamiento, tolerante a mancha- } \\
\text { do de grano }\end{array}$ & $\begin{array}{l}\text { Sistemas tecnifi- } \\
\text { cados Chinandega }\end{array}$ & $\begin{array}{l}5.150^{4} \\
(4.827)\end{array}$ & $\begin{array}{l}3,44 \\
(2,6)\end{array}$ & $\begin{array}{l}0,75^{4} \\
(0,34)\end{array}$ \\
\hline $\begin{array}{l}\text { INTA } \\
\text { DORADO }\end{array}$ & Indica & Alto potencial de rendimiento y rango de adaptación & $\begin{array}{l}\text { Sistemas manua- } \\
\text { les, Siuna }\end{array}$ & $4.500^{5}$ & n. d. & n.d. \\
\hline 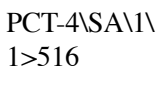 & \apónica & $\begin{array}{l}\text { Muy precoz, apto para una siembra en época de pos- } \\
\text { trera, regularidad de rendimientos. }\end{array}$ & $\begin{array}{l}\text { Sistemas tecnifi- } \\
\text { cados o manuales } \\
\text { zonas del Pacífico }\end{array}$ & $2.231^{6}$ & 3,06 & $0,74^{6}$ \\
\hline
\end{tabular}

1 Promedio de cinco ensayos de validación en finca de productores conducidos en el departamento de Jinotega en 2005; entre paréntesis dato de la variedad del productor.

2 Resultados promedios para cinco ensayos PVS manejados en Wiwili, Bocay y Yali (Jinotega) en 2003 y 2004; entre paréntesis promedio de la variedad del productor.

3 Promedio de tres ensayos de comprobación en finca de productores manejados en el sitio de Belén (Rivas) en 2004 y 2005; entre paréntesis promedio de la variedad del productor (INTA N-1 local).

4 Promedio de tres ensayos de comprobación en estación experimental (2) y finca de productores (1) manejados en la zona de Chinandega en 2004 y 2005; entre paréntesis promedio de la variedad comercial INTA N-1.

5 Promedio de 20 ensayos de validación en la zona de Siuna

6 Promedio de nueve ensayos de validación en finca de productores sembrado en la época de postrera en tres zonas de la región del Pacífico en 2005; Aquí no se presentan datos de la variedad del productor porque el $80 \%$ de los A/E no disponían de una variedad precoz apta para sembrar en época de postrera.

menos organizados y entrenados para manejar experimentos y trabajar con investigadores ${ }^{22}$. Igualmente la

\footnotetext{
22 Ejemplo de los avances del proyecto obtenidos en el sitio de Chinandega comparado a los del sitio de Belén. También podemos hacer la comparación con las actividades FP sorgo conducidas en dos sitios del departamento de Madriz (Unile y Cayantu), donde el proyecto trabajó con grupos de productores previamente organizados y capacitados en experimentación campesina por el proyecto UNICAM/INSFOP: en estos dos sitios, los avances del proyecto fueron aun más rápidos en términos de variedades en fase de validación, de capacidad adquirida por los productores para establecer y manejar los ensayos (incluyendo la toma de datos) y para producir semillas (Trouche et al. 2006).
}

elección de utilizar diversas fuentes de germoplasma ofreciendo una amplia diversidad genética ha sido pertinente. La relevancia de los métodos aplicados para manejar los trabajos de fitomejoramiento participativo (PPB) será analizada en otra publicación.

La interacción fuerte entre los grupos de agricultores y los investigadores, buscada desde las primeras etapas del proyecto, ha permitido definir de modo más preciso y a veces con un carácter novedoso (siembra de arroz en época de postrera) la diversidad de los objetivos de mejoramiento a atender. 
Al nivel operativo, los dispositivos de investigación han demostrado ser bastante eficientes; al contrario se debe subrayar las dificultades en la aplicación de mecanismos de concertación y toma de decisión compartida, en los cuales todos los actores tienen un papel determinante. Asimismo, en dos de los sitios, la ONG u OP socio no asumió plenamente el papel que debía asumir y esto ocasionó atrasos en la secuencia de las actividades, pérdidas de algunos experimentos, participación irregular de los productores en los talleres y sobrecarga de trabajo para el equipo de investigación ${ }^{23}$. Esta evolución de responsabilidades es necesaria; así el proyecto evolucionaría de una situación donde es fuertemente guiado por el equipo de investigación (con la fragilidad que eso implica) a una investigación co-manejada por los usuarios y los investigadores, que podría ser calificada de fitomejoramiento en partenariado.

Además, las lecciones aprendidas de este proyecto nos convencen de la importancia de:

- Elegir las instituciones de apoyo técnico sobre la base de su estabilidad y permanencia de acciones en el sitio y la perennidad de sus financiamientos tanto como de su capacidad técnica.

- Tomar el tiempo y los recursos necesarios para dar al colectivo de participantes las capacitaciones necesarias, identificadas al inicio y durante el proyecto, relativas al papel y tareas que deben asumir.

- Entrenar lo más pronto posible a los A E para que puedan establecer y manejar ellos mismos los ensayos con un apoyo mínimo de los técnicos.

- Utilizar diseños experimentales más específicos y flexibles para los ensayos en fincas de productores y métodos estadísticos adaptados al conjunto de datos generados (cuantitativos y cualitativos).

23 Dilema mayor aún que por escasez de recursos, los investigadores deben en el mismo tiempo: i) continuar el trabajo de creación de nuevos materiales, ii) analizar la multitud de datos generados por el FP, redactar los informes y publicaciones, iii) planificar las actividades en colaboración con los productores y socios, iv) preparar e impartir los talleres de evaluación-selección y retroinformación, v) buscar fondos, vi) insertarse en redes de trabajo regionales e internacionales.
- Las otras lecciones más específicas del fitomejoramiento del arroz que podemos resaltar de este trabajo son:

Las tres líneas CT 15944, que fueron las más promisorias para los sistemas de cultivo manual en San Dionisio, Siuna y Belén, no fueron seleccionadas por los productores en el momento de la primera evaluación participativa del vivero fuente realizada en 2003 en Chinandega ${ }^{24}$ pero fueron "rescatadas" por el fitomejorador con base en los datos agronómicos y postcosecha. Esto demuestra la importancia de la elección del sitio en donde se realiza la primera evaluación de los viveros de germoplasma introducido, y de forma más general, la necesaria complementariedad entre los datos medidos y las apreciaciones de los fitomejoradores y los productores para tomar las mejores decisiones en este tipo de investigación.

Varias líneas seleccionadas en este proyecto presentan buenos resultados y tienen buena aceptación de los productores en diferentes ambientes y sistemas de cultivo ${ }^{25}$ : este resultado nos indica que la estrategia PVS no genera solamente cultivares de adaptación específica sino también cultivares de adaptación geográfica bastante amplia.

\section{Perspectivas}

Para el equipo de investigación, los retos a enfrentar a corto plazo son varios: i) ¿Cómo manejar la fase de difusión de las variedades procedentes del trabajo FP (niveles locales y regionales)?, ii) ¿Qué sistemas descentralizados de producción de semilla serán posible desarrollar para que los pequeños productores logren un

\footnotetext{
${ }^{24}$ A pesar que la mayoría de los productores de arroz de la zona de Chinandega están tecnificados, participó en la evaluación de este vivero un grupo de cuatro productores produciendo arroz bajo sistemas manuales.

25 Además de ser las de mayor productividad en el sitio de Belén (ver Cuadro 5), las líneas CT 15944-10-4-3-3 y CT 15944-10-43-1 se clasifican también entre las cinco líneas de mayor rendimiento en los sitios de San Dionisio y Siuna, superando en promedio a la variedad comercial testigo de entre $+15 \% \mathrm{a}+18 \%$; la línea CT 11231-2-2-1-3-M-4-5-1-M se encuentra entre los cuatro mejores materiales para el rendimiento en los sitios de Chinandega (sistemas tecnificados) y Siuna y San Dionisio (sistemas manuales). Además, estas tres líneas tienen una frecuencia de selección por los productores superior a 0,6.
} 
acceso rápido y seguro a las semillas de los cultivares seleccionados?, iii) ¿Cómo y quién podrá atender los temas de investigación en agronomía solicitados por los productores?, iv) ¿Cómo obtener un financiamiento permanente para sostener las diferentes etapas del proceso (investigación, formación, difusión)?

Sin embargo, el punto clave es la organización de un sistema de creación y evaluación permanente de los nuevos materiales genéticos de arroz, que involucra a los investigadores, las diferentes categorías de productores y los diversos actores de la cadena productiva. Como producto de este proyecto, se ha logrado conformar una red estable y capacitada de instituciones y productores mejoradores de arroz de secano para las zonas de Chinandega y Siuna pero no se logró alcanzar lo mismo en los demás sitios.

Esta experiencia ofrece varias lecciones, aunque no siempre es posible extrapolarlas fuera de su contexto. La estabilidad operacional y financiera de los socios, la motivación y la capacidad de innovación de los investigadores, la calidad y la diversidad del material vegetal disponible al inicio y durante el proyecto, la organización y capacidad técnica de los grupos de productores son factores claves para el éxito de investigación en FP. Asimismo es determinante la capacitación de los actores del proceso, investigadores, técnicos de OP, ONGs y servicios de extensión y productores líderes en su comunidad, sobre el proceso y los diferentes métodos y herramientas del fitomejoramiento participativo. La combinación pertinente de estos diversos factores es la garantía de durabilidad y eficiencia de esta investigación.

\section{AGRADECIMIENTOS}

A todos los productores y productoras experimentadores, evaluadores y mejoradores quienes han participado en esta investigación en los diferentes sitios. También agradecemos a los responsables y técnicos de SERVITEC, CIAT San Dionisio, INTA Siuna, UCA Siuna, UNAG/PCaC Siuna y NITLAPAN quienes han apoyado de una manera u otra en la ejecución de este proyecto.

\section{LITERATURA CITADA}

CHATEL, M.; OSPINA, Y.; RODRÍGUEZ, F; LOZANO, V.H. 2003. Mejoramiento poblacional y obtención de líneas de arroz para el ecosistema de sabana. In: Guimarães, E.P. ed. Mejoramiento poblacional, una alternativa para explorar los recursos genéticos del arroz en América Latina. CIAT, Cali, Colombia. p. 253-269.

HOCDÉ, H.; LANCON, J.; TROUCHE, G. 2002. La sélection participative: impliquer les utilisateurs dans l'amélioration des plantes. Actes de l'atelier organisé les 5 et 6 septembre 2001 á Montpellier, France. CIRADMICAP, 148 p.

HOCDÉ, H. 2006 Fitomejoramiento participativo de cultivos alimenticios en Centro América: panorama, resultados y retos. Un punto de vista externo. Agronomía Mesoamericana. Agron. Mesoam. 17(3): 291-308.

INTA (Instituto Nicaraguense de Tecnología Agropecuaria). 2000. Guía Tecnológica 2. Cultivo de arroz. Managua, Nicaragua, 19 p.

MAGFOR. 2005. Informe anual 2003-2004. Encuestas Agropecuarias de la Dirección de Estadísticas del Ministerio Agropecuario y Forestal de Nicaragua. (en línea). Managua, MAGFOR. Consultado 12 abril 2006. Disponible en: http://www.magfor.gob.ni/ servicios/ servicios.html.

MARTINEZ, C. P.; TOHME, J.; LOPEZ, J.; BORRERO, J.; Mc COUCH, S.R.; ROCA, W.; CHATEL, M.; GUIMARAES, E. 1998. Estado actual del mejoramiento del arroz mediante la utilización de especies silvestres de arroz en CIAT. Agronomía Mesoamericana (CR) 9(1):10-17.

OXFAM, 2005. El arroz se quemó en el DR-CAFTA. Informe 68 de Oxfam. 52 p. (en línea). Boston, Oxfam América. Consultado 10 mayo 2006. Disponible en: http://www.oxfamamerica.org/es/noticias/publicaciones.

RIZO B. H.; PARRALES D. E., 2003. Análisis de las oportunidades de mercado y potencialidades productivas del arroz en el Municipio de Siuna. Tesis de Ingeniero. 
Universidad Centroamericana (UCA). Facultad de Ciencia, Tecnología y Ambiente. Carrera de Ingeniería en Sistemas de Producción Agropecuaria. 61 p.

ROSAS, J.C.; GALLARDO, O.; JIMENEZ, J. 2003. Mejoramiento genético del frijol común mediante enfoques participativos en Honduras. Agronomía Mesoamericana (CR) 14(1):01-09.

TROUCHE, G.; CHOW WONG, Z.; CHATEL, M. 2003. Annual report 2003 for rice and sorghum participatory plant breeding in Central America. 15 p., 12 tab. 2 fig.

TROUCHE, G.; HOCDÉ, H. 2006. Ficha método de fitomejoramiento participativo: Método de evaluación participativa de variedades en campo usando los criterios de los agricultores. (en línea). CIRAD-INRA, Monpellier, Francia. Consultado 25 mayo 2006. Disponible en: http://fitomejoramiento-participativo. cirad.fr/

TROUCHE, G.; HOCDÉ, H.; AGUIRRE S. 2006. Sélection participative des sorghos au Nicaragua: approche et dispositifs. In: Lançon J.; Weltzien E.; Floquet A. eds.
Gestion du partenariat dans les projets de sélection participative. Proceedings de un taller de capacitación organizado del 14 al 18 de marzo del 2005, Cotonou, Benin. CIRAD, INRAB, Cooperation Francaise. En prensa.

VALES, M. 1987. La résistance durable: cas de la pyriculariose du riz. II. Amélioration variétale de la résistance durable. Agronomie Tropicale 42(2): 112-121.

VOM BROCKE, K.; TROUCHE, G.; OUÉDRAOGO, I.; SINGBÉOGO, J.; KABORÉ, R.; BARRO, C. 2003. Evaluation of data from participatory selection in segregating material of sorghum in two areas of Burkina Faso. In: Proceedings of the workshop of Quality of Science in Participatory Plant Breeding, September 30-October 4 2002, Roma, Italy. (en línea). PRGA/ CGIAR. Consultado 8 feb. 2006. Disponible en: http://www.prgaprogram.org/

WITCOMBE, J.; JOSHI, A.; JOSHI, K.O.; STHAPIT B. 1996. Farmer participatory cultivar improvement. I. Methods for varietal selection and breeding and their impact on biodiversity. Experimental Agriculture, 32: 445-460. 